\title{
Mobile Applications Integrated to Modeling and Simulation: An Accessible Tool for Strategic Stock Management of Clinical Supplies
}

\begin{abstract}
Adalberto Valadares Lourenço Pereira ${ }^{1}$, Elioenai R. da Silva ${ }^{2}$, Lucas E. de Oliveira ${ }^{3}$, Anna Tércia de Azevedo Almeida ${ }^{4}$, Daniela Da Guarda Ribeiro ${ }^{5}$, Fernanda de Paula Vitor $^{6}$, Gabriela Silva Rangel ${ }^{7}$, Ilia Helena Teixeira Kumaira ${ }^{8}$, Lara Goulart Pereira9, Lavínia Ribeiro Tavares ${ }^{10}$, Raquel Bretas de Assis Hallack ${ }^{11}$, Braulio R. G. Marinho Couto $^{12}$, Diva Souza e Silva Rodrigues ${ }^{13}$, Luiz Melk de Carvalho ${ }^{14}$, Flávio Henrique Batista de Souza ${ }^{15 *}$

Centro Universitário de Belo Horizonte - UNIBH, Brazil
\end{abstract}

flabasouza@yahoo.com.br

\begin{abstract}
In COVID-19 times, expenditures and investments on health clinics must be rigorously evaluated so that there are no excesses and shortages. Thus, a structure that integrates some of the most used technologies today, such as: mobile applications, cloud computing, data analytics, modeling and simulation to discrete events; is presented in this paper as an accessible tool for health clinics. Real data from six months of analysis in a medium-sized health clinic situated at a university in Belo Horizonte, guided the demand, design, implementation and testing of the developed tool. As results, the following items were obtained: a mobile application for inventory management, with authentication control (of teachers, students and others involved) and access levels; a cloud computing structure for data consolidation and feasibility for data analytics; and a modeling and simulation structure, based on stochastically timed Petri nets (STPN) for the evaluation of continuous or fixed supply replenishment contracts. As contributions, this solution includes: consolidation of input stock data; smart spending management; in addition to the ability to simulate scenarios to assess the effects of contracting replenishment of inputs in the health area. Questions like: what is the best form of replacement contract? is it better to buy an entire stock or stipulate continuous replenishment? These and other questions can be answered by the simulator developed via STPN helping clinics and hospitals of different sizes on the management of their supplies.
\end{abstract}

Keywords: Mobile Applications, Stochastic Timed Petri Net, Clinical Inventory Management, Cloud Computing, Health Tools

\section{Introduction}

In order to obtain maximum performance when managing an organization, it is necessary to carry out a rigorous inventory management process, which is one of the main points of attention 


\section{$3^{\text {rd }}$ International Conference on Research in SCIENCE, ENGINEERING AND TECHNOLOGY}

in a company due to the consequences arising from failures throughout its execution. A high level of experience and maturity is required and the problems in this management have affected companies of the most varied segments and sizes. Inventory management aims to define a balance between the required demand and materials needed to support this service, ensuring market stability (Ramos et al., 2015). In addition, it contributes to the growth of the company and to the reduction of business costs (Batista et al., 2020).

Once the level of complexity of the processes that guide a hospital clinic is understood, there is a latent need to apply several logistical concepts to guarantee an efficient service, minimizing investments and maximizing the financial return and customer satisfaction. (Ronan et al. 2019).

Referencing a hospital institution, the consequences can be more aggravating, considering that the lack or excess of medicines, materials used to provide care and surgical equipment affect not only the company, but the employees, and especially the satisfaction of their patients (Pestana et al., 2020). In addition to the above, a serious aggravation permeates society: the need to restructure companies economically affected by COVID-19, in the year of 2020. These companies must use maximum intelligence to manage their businesses, since several companies closed the doors because of the lack of capital to survive in this difficult time. In addition to those that, even if they are surviving, must fully exploit the demands for smart spending, as their financial resources are compromised.

Given this reality, a case study was carried out on existing processes during inventory management. It is subdivided into several other interdependent processes, each with its welldefined objectives: inventory checking; budget period; order planning; receiving and checking; storage process and in some moments storage of stock; product identification and classification; systemic registry; shipment and carry out the discharge and consumption process by the end customer.

In response to the scenario presented, the general objective of this article is to present intelligent solutions with the capacity for efficient decision making, providing greater assertiveness during the entire inventory management process of hospital companies. With specific objectives, this work aims to: outline the stock process of low, medium and large hospital institutions; develop and implement a solution based on mobile technologies, cloud computing and modeling and simulation that serves this segment; evaluate the return on investment that the tool can bring to the organization.

Batista et al (2020) demonstrated a solution that combines the concepts of cloud computing, mobile applications and modeling and simulation, for the stock control of a medium-sized orthopaedic clinic. This work represents an expansion of this methodology and is justified by the presentation of a scalable solution in terms of company size, in addition to a manageable system of administration and strategic management.

\section{Materials and Methods}

\subsection{Development Concepts}

For the development of the proposed solution, the following concepts were used: 


\section{$3^{\text {rd }}$ International Conference on Research in SCIENCE, ENGINEERING AND TECHNOLOGY}

Mobile Applications: according to Batista et al. (2020), software that can be allocated on mobile devices is highly accepted by contemporary society. They must be robust, but simple, because they are aimed at devices with limited computational resources.

Cloud Computing: represents a connection resource and provision of structure over the internet. According to Batista et al. (2020), this concept is widely used in the context of industry 4.0, enhancing: data storage, availability of resources and accessibility.

Stochastically Timed Petri Nets: according to Lisboa et. al (2019), the basic elements that guarantee the definition of a Petri net, are multipurpose and to a large extent can be interpreted freely. These elements are as follows: Places $P$ (white background circles where resources are allocated); $T$ transitions (bars representing the occurrence of an event); $A$ arcs that interconnect $T$ and $P$ and vice versa. The "trigger" of a transition represents the consumption or attribution of a token (the black circles, inside the places and represent a resource). The number of chips is represented by an $x$ mark. The triggering of a transition can obey a time that is not deterministic, but stochastic, which can represent a greater degree of simulation reality. Figure 1 shows an example of this type of Petri net.

Source: Lisboa et. Al, 2019.

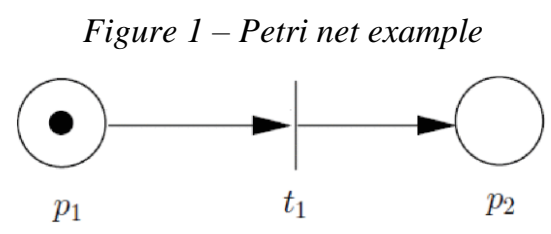

\subsection{Research Methods}

During the period from March to July of 2019, data were collected to analyse the process of using the stock of materials that are the inputs for practical clinical care classes. The objective was to evaluate, not only how was the current the process, but to evaluate the demand for an automated system to control information.

At first, the seven main products, which were judged to be the most used by the respective interest group and registry, were analysed during the mentioned months for an understanding of the demand of these products in a small / medium-sized clinic. The demand that was raised is based in a system with capacity to perform registrations, deletions and updates of data of users, doctors, materials, suppliers, types of materials, and with separation of the access of each user through groups of permissions.

The implementation process consisted of three stages: the first consists of defining tools to generate the mobile application, thus resulting in a systemic structure; the second was the implementation of the mobile structure; finally, intelligent management structures based on statistical analysis for strategic management (data analytics) and demand simulators based on stochastically timed Petri nets were developed. They were used to simulate demands for continuous or fixed contracts, to answer questions such as: is there a lack of input? what is missing? When? so that more advantageous contract strategies can be developed for the clinic (consequently for the university). 


\section{$3^{\text {rd }}$ International Conference on Research in SCIENCE, ENGINEERING AND TECHNOLOGY}

11-13 March, 2021

Oxford - United Kingdom

\section{Results}

\subsection{System Structure}

In the first instance, this solution has the capacity to record the receipt of any goods or items used by the organization. It has the computational capability to identify and register the data in a database to guarantee the access of the stock information in real-time. Figure 2 shows an organization of the developed structure. The solution performs the inventory write-off process, according to its respective information, notifying in case any product needs a replacement action, in view of the business rule determined by the customer. Through the Cordova platform, a solution that can be accessed via the web or mobile structure, it serves as a tool for registration, as well as for applying quick queries to the stock, so that all available materials, materials to be replenished, and consumed are presented.

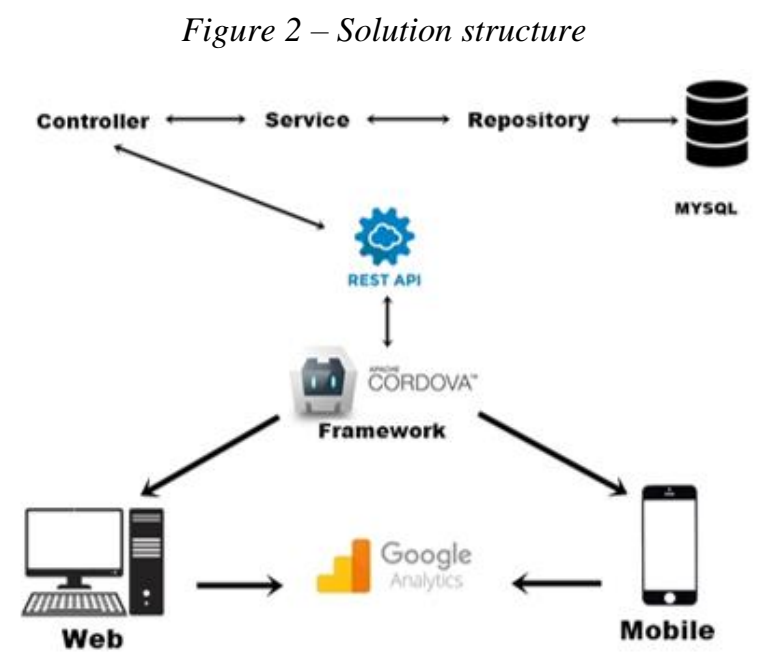

Source: Authors, 2020

Such a structure is connected to a database system responsible for the consolidation of the information. During the service in the organization, the application (web / mobile) is responsible for several functionalities: show the products to users for consultations and to access detailed information about them; management reports of the consumption; order history; and balance sheets with a period defined by the responsible user.

\subsubsection{Mobile Structure}

For the processes involved in using the application for stock management, user control and user permissions, some screens were created to guarantee a high usability for the user. The application has interface for login, reports updated in real time, registration of inputs and medicines, as well as their categorization, and records of new users, always aiming to obey the hierarchy of access based on the profile used at a given time.

To exemplify this scenario, Table 1 shows the screenshots of the different screens of the mobile interface. Figure 3 shows an image of the system diagram and Figure 4 shows a 


\section{$3^{\text {rd }}$ International Conference on Research in SCIENCE, ENGINEERING AND TECHNOLOGY}

flowchart of the use of the application. Some premises are maintained for the functioning of the mobile application with respect to its flow and the three types of users present, where:

- The workflow of the system starts at the login screen where the user already registered must enter their email and password. In case the user has no registration, they can register, when entering the system, on the permission group to which the user belongs;

- Student user: the student group user has only the consultation permissions in the system, being able to consult the stock situation and issue reports.

- Administrator user: this group has the consultation permissions that the student has, in addition to registering materials and being able to download the reports.

- Master user: this group of permissions is the highest level in the system, having all the permissions that the previous groups and the only one with the option to register a responsible person.

Thus, with such respected prerogatives, the flow of use of the application begins. When the application is started, it will display a screen (Table 1 - Screen 1) that will load its data showing the name and logo.

Figure 3 - Solution structure

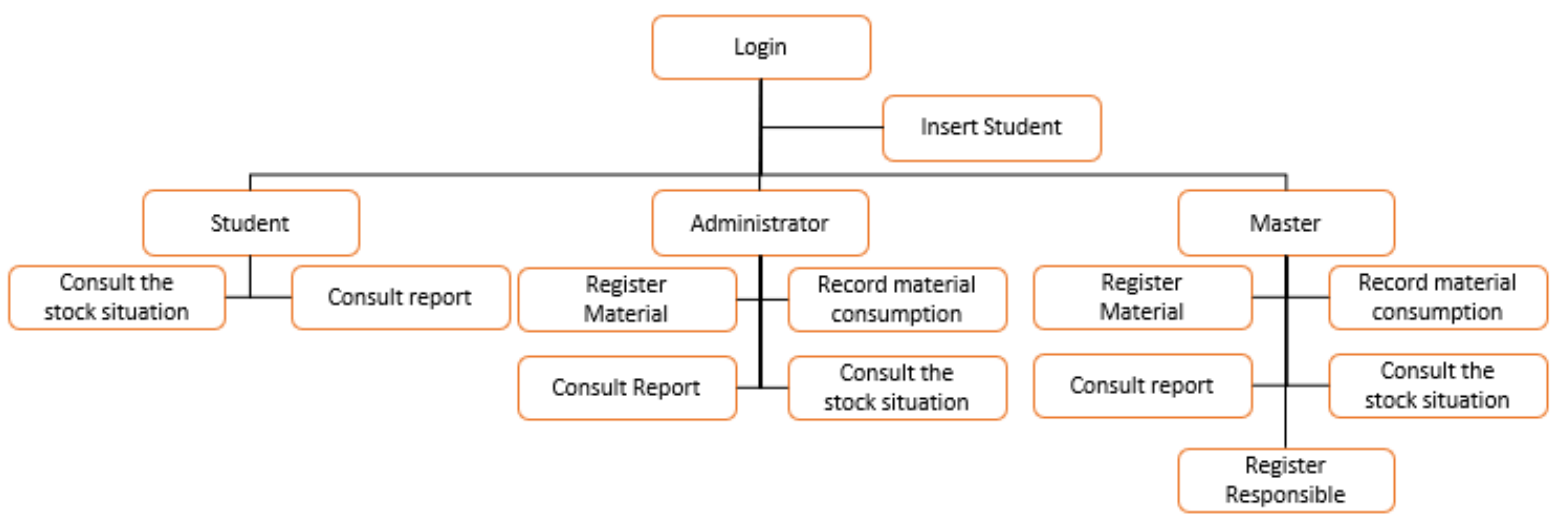

Source: Authors, 2020 


\section{$3^{\text {rd }}$ International Conference on Research in SCIENCE, ENGINEERING AND TECHNOLOGY}

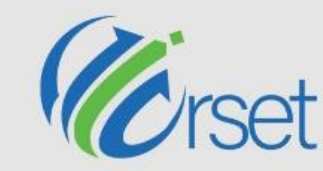

11-13 March, 2021

Oxford - United Kingdom

Figure 4 - Utilization Flowchart

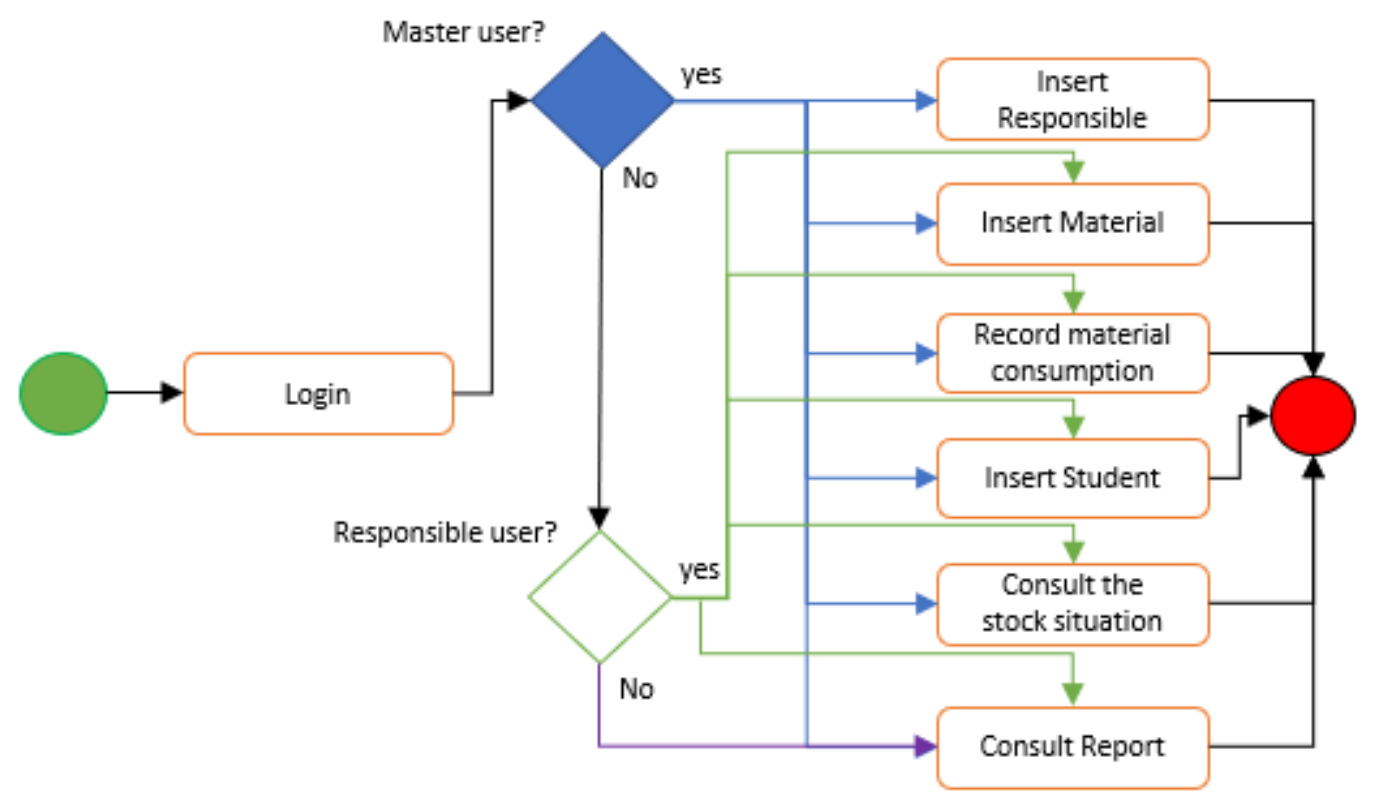

Source: Authors, 2020

After completing the loading process, it will be changed to a screen responsible for access control in the tool (Table 1 - Screen 2), which is done through an RA (Academic Record) or registration or through a user in the standard adopted by the company. By clicking on the "Enter" button at the bottom of the same screen, those informed will be submitted to a validation step, through the records in the database. After completing this validation, if they are correct, the dashboard (Table 1 - Screen 3) that is enabled for the user in question will be displayed based on the user's permissions. If the user does not have a registration, on the same screen a link is found to the screen responsible for registering data in the system via the database (Table 1 - Screen 2), at this moment the user is asked to enter their identification data, which are Full Name, user, Institution to which it belongs, e-mail linked to the company and a password. After that, the registration will be inserted in the database observing the data types of each field. 


\section{$3^{\text {rd }}$ International Conference on Research in SCIENCE, ENGINEERING AND TECHNOLOGY}

11-13 March, 2021

Oxford - United Kingdom

Table 1: Screenshots of the application screens

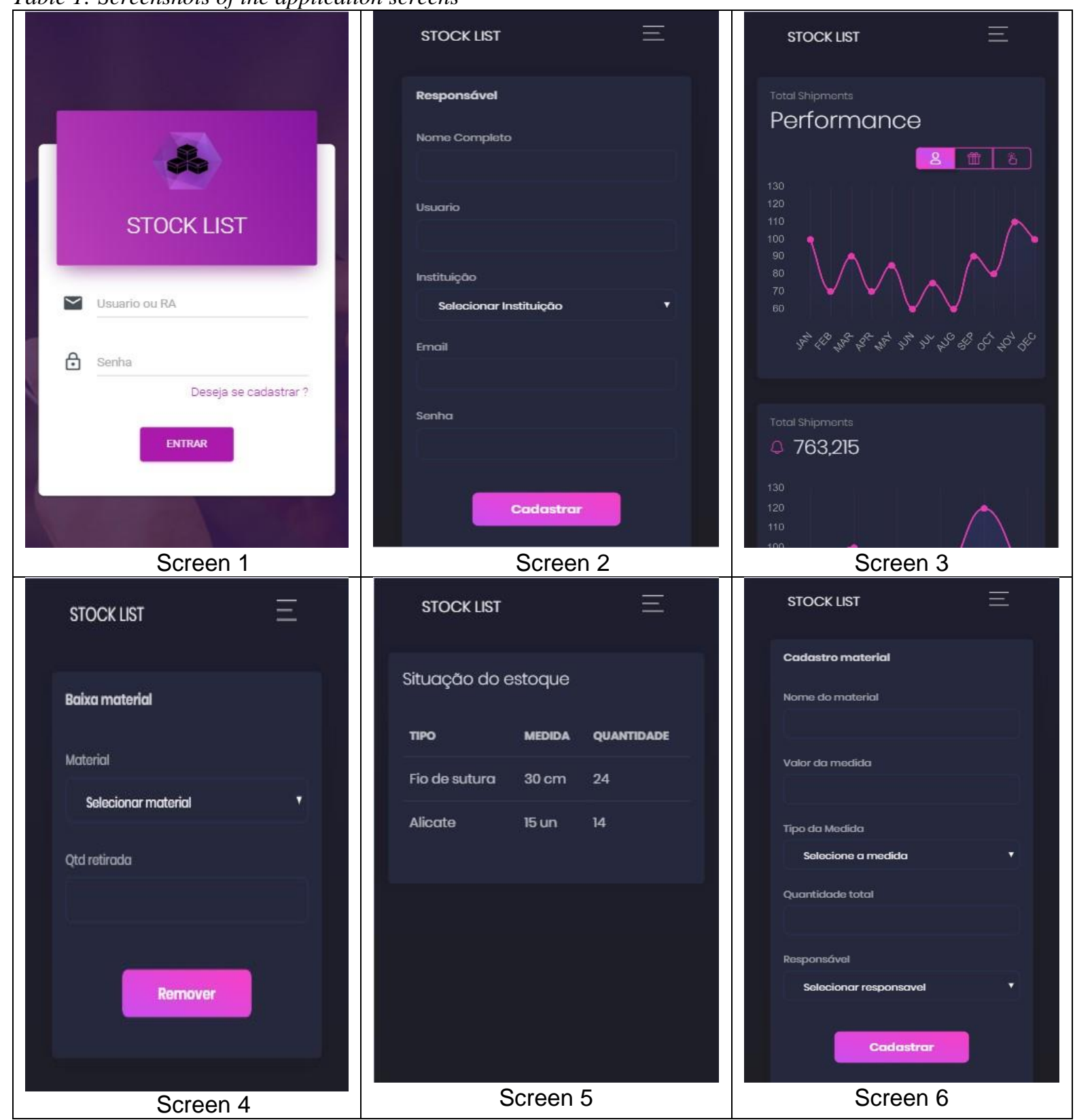

Source: Authors, 2020

At the end of the login, the report screen will be presented to the user to monitor the amount of medicines, products, and materials available in stock and various information such as Name and specific characteristics of each product when necessary (Table 1 - Screen 5 ). The manager or responsible person is also allowed to register new products on a specific screen (Table 1 Screen 6). On this screen, the user is asked to inform the Material Name and Measurement Value that relates to specific information such as the thickness of suture threads.

In the Type of Measure field, it is used to inform the unit of measurement of the product in question. Depending on the product type its material description can be based in metrics such 


\section{$3^{\text {rd }}$ International Conference on Research in SCIENCE, ENGINEERING AND TECHNOLOGY}

11-13 March, 2021

Oxford - United Kingdom

as milligrams, tablets, among others. In the total quantity field, the gross quantity of the products is informed. Then, the name of the person responsible for accessing the system and performing the registration is requested. This information is displayed in a component called Listbox (a kind of list), which is responsible to automatically fill the user information based in their login data, and thus preventing this information from being misinformed. Thus, with a screen to be used at the time that the material will be requested for service, it is necessary to inform which material is desired and its quantity and write it off in stock (Table 1 - screen 4). For inputs that have their own characteristics, as previously said the suture thread, the information will be displayed automatically to ensure assertiveness when requesting. An additional feature is the ability to export data in ".csv" format to allow posterior analysis of the data in consolidated software on the market (Microsoft Excel® for example).

\subsection{Statistical Analysis Structure}

An analysis tool that is incorporated into the system is the dashboard of consumed materials, which is presented on the initial screen to show the user the real-time situation of the stock, thus allowing continuous monitoring (Table 1 - Screen 3). As already mentioned, there is a database in which it is possible to consolidate the information as the solution is used. With this, a resulting dashboard will be able to guide the manager about the demands that are present in the clinic. In parallel to the mobile application development a data collection was carried out from March of 2019 to July of 2019, checking the main materials that are consumed during classes, namely: tongue, string, nylon suture, scalpel blade, sterile gloves, procedure gloves and pieces of meat.

In this research, the focus was kept on data that is consolidated by the mobile application. Its objective is that real data were inserted to prove the effectiveness of the hypothesis that information from the dashboard of the developed system really consolidated coherent information and that it could be evaluated graphically. Some questions were asked to illustrate a moment of analysis by the responsible manager, considering a group of students, the mentioned inputs and the period considered: How much is spent per month for each material? What is the average expenditure per student? What is the total consumption of these inputs? What is the average monthly expenditure for each input?

These are basic questions, but with great relevance for any business, regardless of their size. The graphs in figure 5 were created based on the data collected and showing the monthly consumption of materials, the average monthly consumption, the total consumption based on each of the materials and the average consumption of each material. It should be noted that the graphics were assembled in a dimensionless way (i.e. only quantitative data) for a first quantitative analysis of the previous-mentioned materials.

Despite a dimensionless analysis, it was possible to see the ability to answer the questions assessed as elementary to a business. Dimensional measurements may not be useful for statistical analysis, but they can be used in mathematical tools, such as Petri nets (to be demonstrated in section 3.3) where units can be abstracted.

In addition, as described in section 3.1.1, units can be inserted, if not present in the application. For a more detailed analysis, evaluating by dimensional units (unit, centimetres, meters, litres, etc.) two aspects were evaluated: products that can be consumed by units (Figure 


\section{$3^{\text {rd }}$ International Conference on Research in SCIENCE, ENGINEERING AND TECHNOLOGY}

11-13 March, 2021

Oxford - United Kingdom

6) and products consumed by the unit of meters (Figure 7). Note the consistency in both analyses (dimensional and dimensionless), where the consolidation of information was not an obstacle to the structure developed.

Figure 5 - Consumption graph

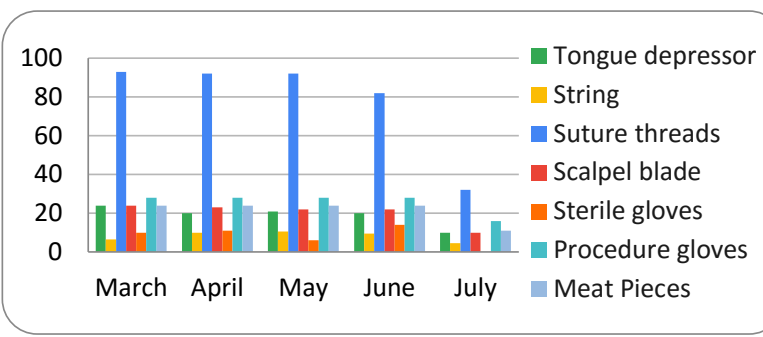

(a) Consumption of materials per month

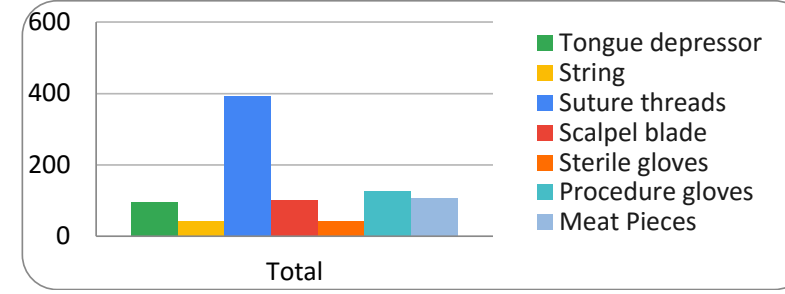

(c) total materials (March to July)

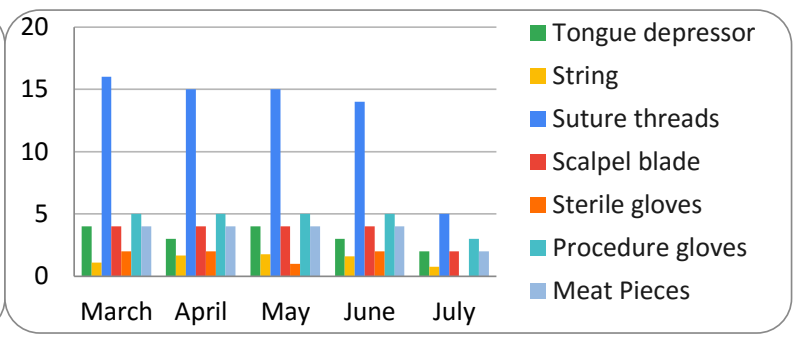

(b) total materials per month for each student

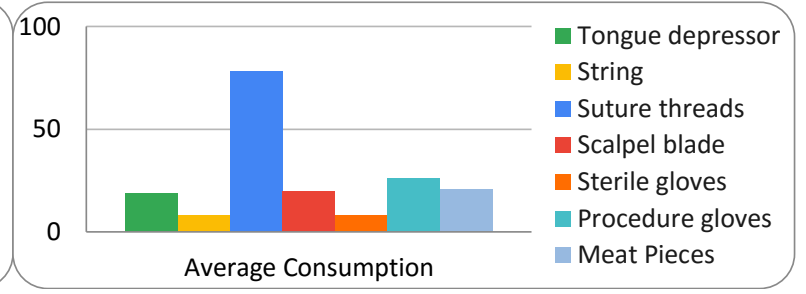

(d) monthly average for materials

Source: Authors, 2020

Figure 6-Graph of consumption of elements measured by units

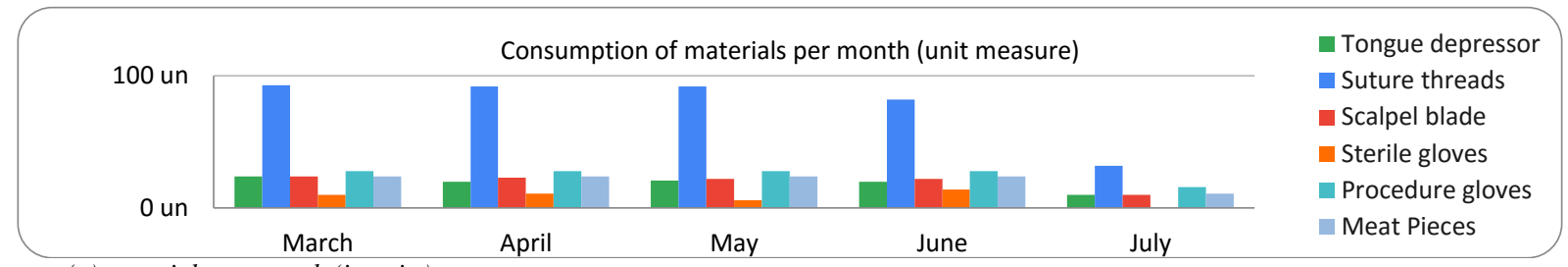

(a) materials per month (in units)

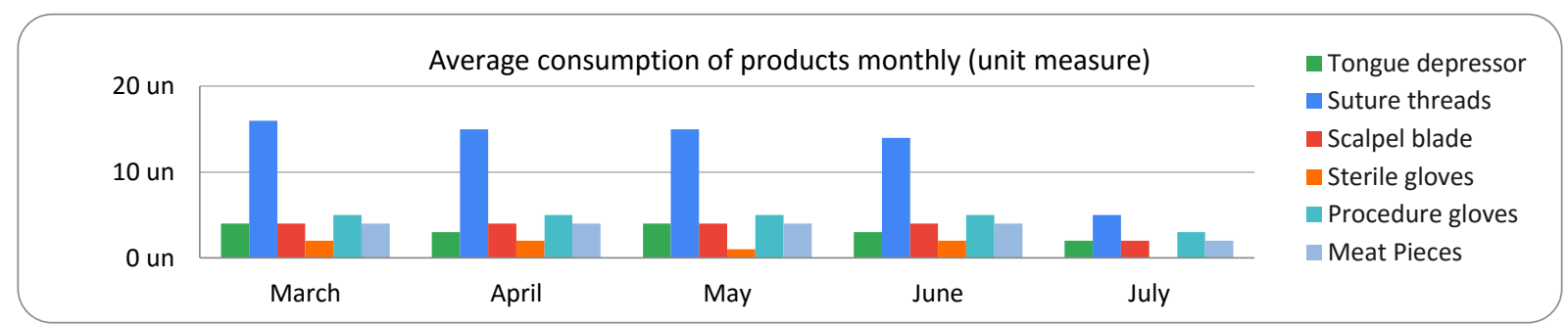

(b) monthly average of each student (in units)

Source: Authors, 2020 


\section{$3^{\text {rd }}$ International Conference on Research in SCIENCE, ENGINEERING AND TECHNOLOGY}

Figure 7 - Graph of total material consumption per month with elements measured by meters
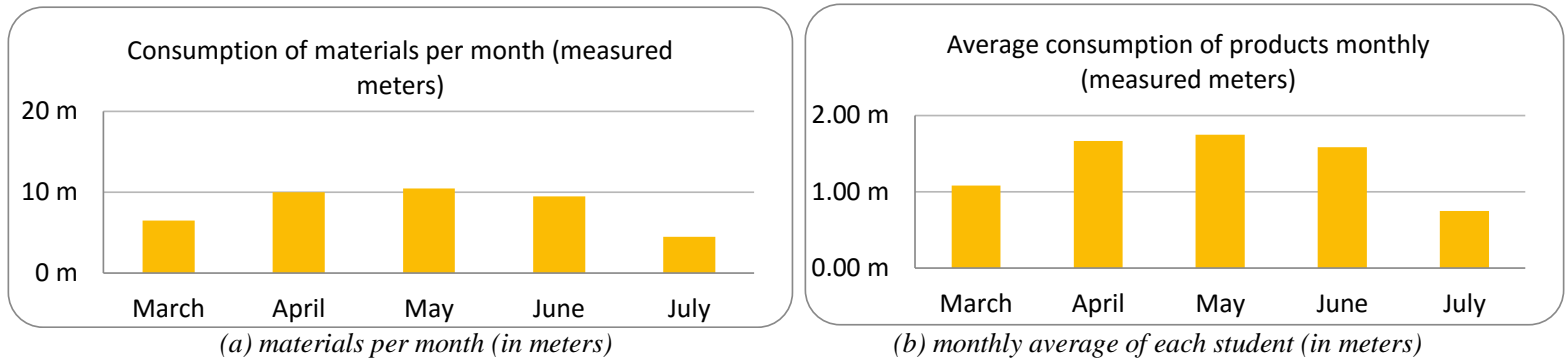

Source: Authors, 2020.

\subsection{Modelling and Simulation Structure}

As the data are consolidated, some viability is obtained and among them the possibilities to guide simulations of scenarios by using tools described in the literature. Thus, an experiment was carried out for modeling and simulation via Petri nets. There were two strands of analysis, considering the amount of materials in stock, the capacity of the stock and the average daily expenditure. The first modeling and simulation contemplates the possibility of continuous supply replenishment contracts (example: every 20 days there is suture replenishment). The second model, on the other hand, considers a closed purchase contract to analyse the availability of materials during the school months. One caveat: all simulations used timed Petri nets. However, through the analysis of the real consumption of the clinic, it was seen that despite the consolidations, the daily use does not obey a constant time. Thus, a timing was proposed that follows an exponential stochastic distribution.

\subsubsection{Simulation of Continuous Contracts}

In this first model analysis, a continuous input was considered. In figure 8, the example of two structures can be seen, each one showing the control of a specific input. The unit of measurement of this Petri net was stochastic in replenishment time and stochastic in spending, with each input having its specific structure. Taking as a reference the first structure, which simulates a replenishment of scalpels, the following scenario was simulated. The transition $T=$ $\{$ Replenish Scalpel\}, has a fixed average, simulating that replenishment takes place every two days after the order is placed. Two premises are placed in check: the order will only be placed if the stock has the capacity to receive the contracted quantity (limited capacity in place $P=$ \{Scalpel Blade (units available)\}, which in this case would be 300 units); the order is placed in a stochastic time based on the delivery of the supplier, simulating delays or advance of deliveries by the suppliers (in this example, $T=\{$ Replenish Scalpel $\}$ had a stochastic average time of $\mu=2$ days). The contracted replenishment (theoretical) is 200 units for each order (represented in arc A between $T=\{$ Replenish Scalpel $\}$ and $P=$ $\{$ Scalpel Blade (units available)\}). 


\section{$3^{\text {rd }}$ International Conference on Research in SCIENCE, ENGINEERING AND TECHNOLOGY}

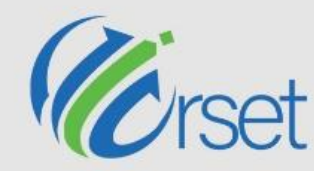

11-13 March, 2021

Oxford - United Kingdom

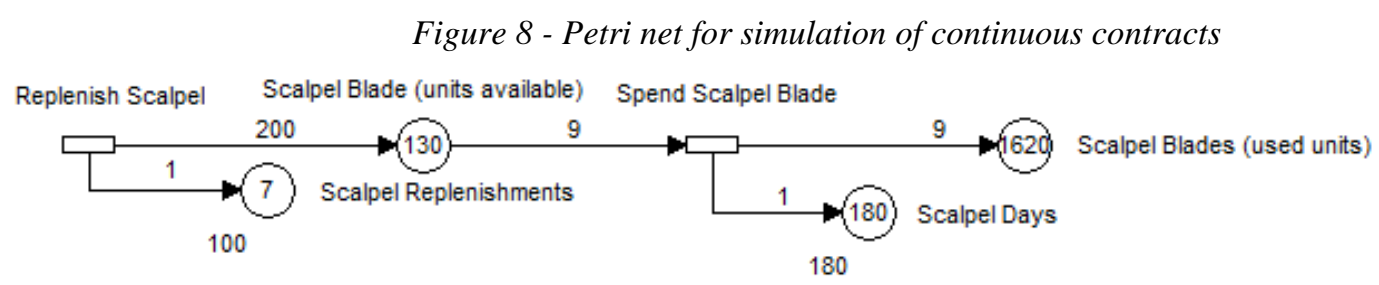

Source: Authors, 2020

Each replenishment is counted in place $P=\{$ Scalpel Replenishments $\}$. In $P=$ $\{$ Scalpel Blade (Units Available)\}, the units that are present in stock are counted. The transition $T=\{$ Spend Scalpel Blade\}, shows an exponential stochastic time of mean $\mu=1$, symbolizing the stochastic analysis that in approximately 1 day, 9 scalpel blades are spent. The quantity of 9 units is provided by the statistical analysis tool - shown in section 3.2, with an average of daily expenses, in whole value, considering a specific period, which can be from months to years. In this case, the 180-day time was simulated, where the place $P=$ $\{$ Scalpel Days $\}$ shows the number of simulated days. A particularity is that the simulation days of each material maintain their particularity, where the analysis of the days of the Scalpel input does not interfere with the analysis days of Suture Threads. In the graph of Figure 9 it can be seen that in a simulation with an initial state of 0 units in stock, the replenishment did not present a breaking point, reaching around 45 to 50 units, making the availability of inputs effective.

Figure 9-Supply Graph

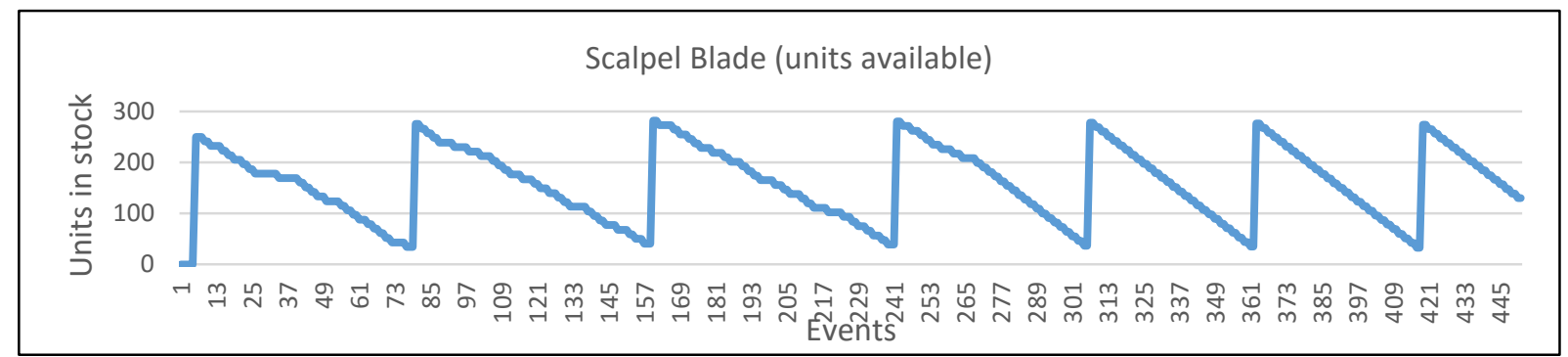

Source: Authors, 2020

A possible strategy to be allocated would be, in case of a reduced budget, instead of 250 units, something between 200 and 220, enabling a renegotiation of values with the supplier. In a simulation with 200 units, it was seen that the number of refills increased in 180 days (from 7 to 9 refills), but with $20 \%$ fewer scalpel units. It is up to the manager to check what is most advantageous.

\subsubsection{Closed Purchase Contract}

Another modeling and simulation was carried out using the Petri net, considering a single material supply contract that should last for one semester. In the case of Figure 10 it was the nylon suture thread and through this simulation it is possible to identify exactly how long the stock will last, when replenishment will be necessary and allows a simulation to be made in order to identify the amount of material that will be requested in a semi-annual contract. 


\section{$3^{\text {rd }}$ International Conference on Research in SCIENCE, ENGINEERING AND TECHNOLOGY}

Every analysis described is weighted by the profile of the clinic. In this model, a fixed, single purchase contract of 480 units of suture thread ( 20 boxes with 24 units each) was first analysed. The presence of this purchase in stock was simulated, represented by the place $P=$ $\{$ Nylon Suture Thread $(U N)\}$, which has a maximum capacity of 900 units. Thus, each month, of the 5 analyzed, gained an analysis module, considering the days of each month (which vary). The weights between the place $P=\{$ Nylon Suture Thread $(U N)\}$ and the initial transitions of each consumption month $T=$ Month 1, Month 2, Month 3, Month 4, Month 5$\}$ have the respective weight of the average expenditure simulated product diary (coming from the consolidation of the use of the application - rounded, since it must be an integer value). Each month is analysed individually, and from the second month onwards, the expenditure of the previous month will always be evaluated, generating a sequential and consistent expenditure. The consumption of each month is simulated and counted in a place called Consumption $x$ Month, which in this case, in the first month will be $P=$ \{Consumption 1st Month\}. For this analysis, a maximum quantity of 600 units was limited (which can be increased or decreased according to the profile of the user).

Also connected is the transition $T=\{$ Month 1$\}$ and the place $P=\{$ Days Month 1$\}$ which requires that each day of the analysed Month 1 should be counted. In this case, the month of March, which was considered Month 1, had a total of 31 days under analysis. A particular issue is present in this type of place in each of the months: if by any chance the stock is not enough for the month, it will inform to the user exactly the day of the respective month that the product will be out of stock (alerting the user about the scarcity input). For instance, if $P=$ $\{$ Days Month 5$\}$, at the end of the simulation and there are 23 cards, on the $23^{\text {rd }}$ of the $5^{\text {th }}$ month analysed it will be the day of lack of nylon sutures. 


\section{$3^{\text {rd }}$ International Conference on Research in SCIENCE, ENGINEERING AND TECHNOLOGY}

11-13 March, 2021

Oxford - United Kingdom

Figure 10 - Petri nets for simulation of closed purchasing contract - simulation of nylon threads

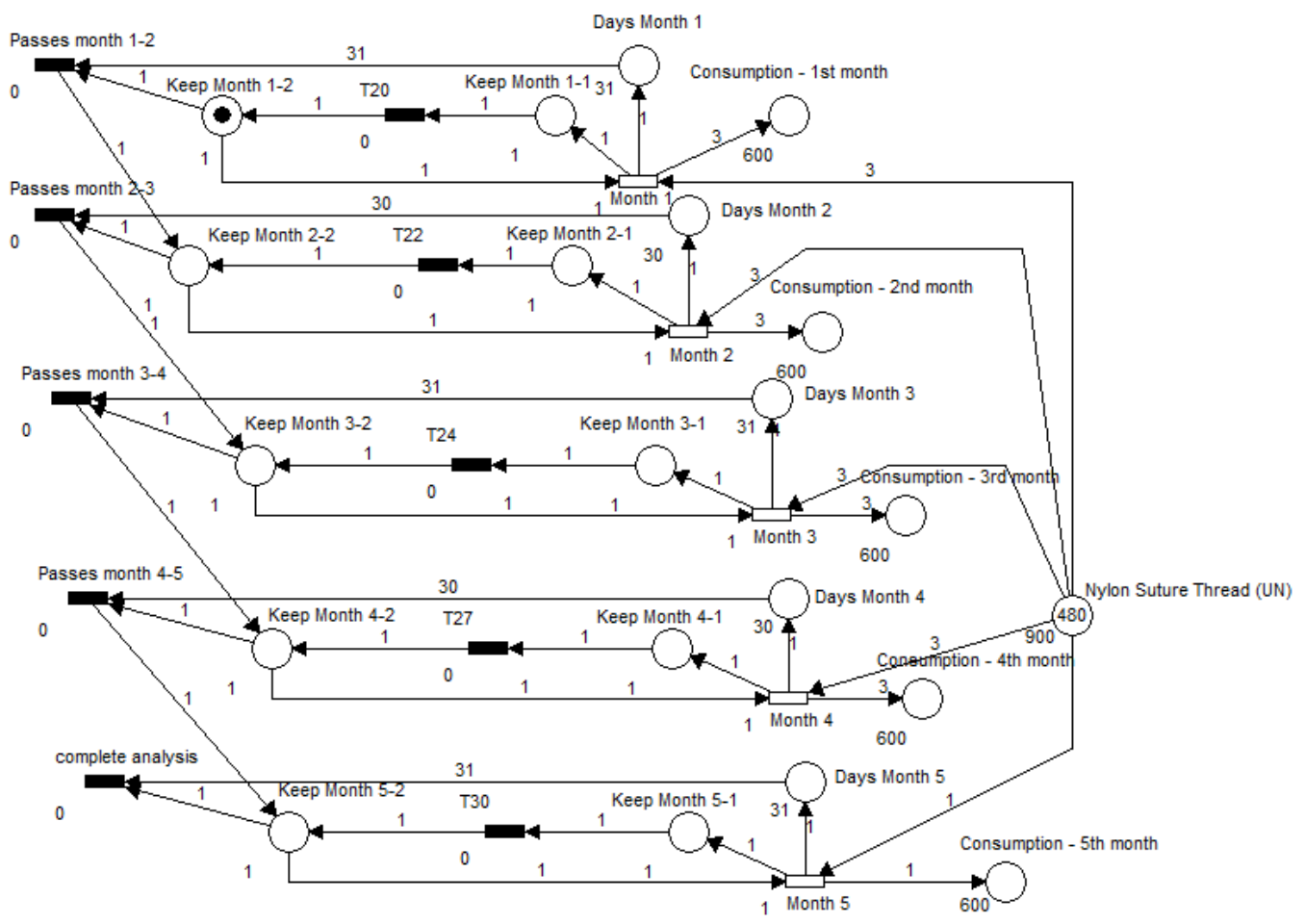

Source: Authors, 2020

Finally, a consistency structure is demonstrated. The transition $T=\{$ Passes month $1-$ $2\}$, which is replicated with each passage of Month, is the transition that allows to move from Month 1 (in this case - March) to Month 2 (April), if and only if , the stock has filled the 31 days of March. The check is double, as it will only fire if there is a card in $P=$ $\{$ Keep Month $1-2\}$ and the number of cards needed between it and $P=\{$ Days Month 1$\}$ (in this case 31 cards, 1 card per day). If these two conditions are met, the next month will be the simulation's turn. This process is repeated until $T=\{$ complete analysis $\}$ (if all months have been met). If it meets every month, at $P=\{$ Nylon Suture Thread $(U N)\}$, the final result is the amount left over from the semester period of the clinic. Simulating the demonstrated structure, in the case of nylon suture thread, in units, consumption was as follows: 1st Month - 93 units; 2nd Month - 90 units; 3rd Month - 93 units; 4th Month - 90 units; 5th Month - 31 units; Full service ?: Yes; Left: 83 units. Each product has its structure, where a greater (or less) number of months can be simulated

\subsection{Comparative Analysis}

As demonstrated, the solution described has not only the ability to guide information about the stock status of a clinic, but it can help universities with a low budget (or not) to objectively control the stock of supplies. In addition, helping to make decisions that can be better targeted. In a brief comparison with works in the literature, it is possible to compare the results obtained with what is available in some articles. As previously mentioned, there was already an example implementation by Batista et al (2020) in an orthopaedic clinic. However, in its modeling and 


\section{$3^{\text {rd }}$ International Conference on Research in SCIENCE, ENGINEERING AND TECHNOLOGY}

simulation, such work does not include the analysis of fixed contracts, only continuous replenishment contracts.

This work contemplates the two contractual issues, with the addition of the analysis of the specific days of lack or attendance of inputs, besides a tool with capability to carry out a data analytics. Souza et al (2019) also presented the modeling and simulation for inventory control of a pharmacy. Although the control succeeds, including simulating a company's "zero day", it is not integrated with a mobile solution, nor with a solution capable of carrying out data analytics, such as the one presented. Thus, it is possible to determine that there was a contribution from the integration between mobile solutions, data analytics, cloud Computing and Petri networks.

\section{Conclusion}

The work demonstrated a viable solution and demanded by the Brazilian (and even worldwide) economic scenario, of tools that help managers in relation to purchasing and inventory decisions. After the occurrence of COVID-19, where it was possible to see a shortage of various materials for input of procedures in several countries, and where not all treatment centres have intelligent tools to assist their operation. The simplicity of the mobile solution, with regard to its usability, allows people, with a medium-low education in technology, to manage their stock of inputs, in addition to being able to evaluate contracts (whether private or government) to manage their inventory.

Finally, modeling and simulation, which guides future spending, in addition to verifying the effectiveness of signed contracts (or under analysis), demonstrates an advance in management strategies, being able to answer questions such as: do I have the necessary amount of inputs? will I have a shortage? If it is not enough, how long will my stock serve? Such questions, in cases of administration of healthcare institutions, can differentiate the form of patient care.

\section{References}

[1] Ramos, E. D., Pettit, T. J., Flanigan, M., Romero, L. and Huayta, K. (2020). Inventory Management Model Based on Lean Supply Chain to Increase the Service Level in a Distributor of Automotive Sector. International Journal of Supply Chain Management (IJSCM), 9(2), 113-131.

[2] Batista, D. T.; Rocha, V. A. R.; De Souza, F. H. B.; Carvalho, L. M.; Mellim, R. D.; Ferreira, T. A. S. (2020). Mobile Applications and Discrete Event Systems: Low Cost Technology to Assist Stock Management in an Orthopedic Clinic. Proceedings of The 10th International Conference On Research In Engineering, Science And Technology, Rome, v. 1.

[3] Ronan, S.; Brown, M. and Marsh, L. (2020). Parents' experiences of transition from hospital to home of a child with complex health needs: A systematic literature review. Journal of Clinical Nursing, v. 29, n. 17-18, p. 3222-3235.

[4] Pestana, M.; Pereira, R. and Moro, S.(2020) Improving Health Care Management in Hospitals Through a Productivity Dashboard. Journal of Medical Systems, v. 44, n. 4. 


\section{$3^{\text {rd }}$ International Conference on Research in SCIENCE, ENGINEERING AND TECHNOLOGY}

11-13 March, 2021

Oxford - United Kingdom

[5] Lisboa, A. C., De Souza, F. H. B., Ribeiro, C. M., Maia, C. A., Saldanha, R. R., Castro, F. L. and Vieira, D. A. (2019). On modelling and simulating Open Pit mine through stochastic timed Petri nets. IEEE Access, 7, 112821-112835.

[6] Souza, F.H.B; Silva, N. N. and Novaes, P. H. N. F. (2019) Ferramenta Baseada em Eventos Discretos Para Gestão De Estoque Em Uma Pequena Empresa Do Ramo Farmacêutico. Proceedings of XXXIX Encontro Nacional De Engenharia De Produção, Santos, v.1. 